\title{
Erratum
}

The order of the authors of the paper "Selenium in poultry nutrition and its effect on meat quality" which was published in WPSJ 67(3): 479-484 was incorrect. It should read:

\section{Selenium in poultry nutrition and its effect on meat quality}

V. STANAĆEV and N. PUVAČA 